\title{
The Ontario Brain Institute: Completing the Circle
}

\author{
Donald T. Stuss
}

\begin{abstract}
The Province of Ontario recognized the pressing need to improve the understanding, diagnosis, and treatment of brain disorders. It also recognized that maximizing the existing strengths through a province-wide integrated approach was a pivotal mechanism. To achieve this, the Province established the Ontario Brain Institute. The goal of this article is to introduce the elements of the Ontario Brain Institute to the neuroscience community: the motivation for establishing it, the philosophy behind its creation, the principles guiding its development, the rapid evolution of its functional structure, the tools available to achieve its vision, and the management structure to ensure success. The singular goal of the Province and the Ontario Brain Institute is a comprehensive system that assures that basic research is embedded in the clinical system and is facilitating product development to accelerate benefits to both health and the economy of health: science with impact.
\end{abstract}

RÉSUMÉ: L'Institut ontarien du cerveau : boucler la boucle. La province de l'Ontario a reconnu le besoin pressant d'améliorer la compréhension, le diagnostic et le traitement des troubles cérébraux. Elle a également reconnu que le renforcement des forces existantes dans une approche intégrée à l'échelle de la province était un mécanisme essentiel. Pour y parvenir, la province a créé l'Institut ontarien du cerveau. L'objectif de cet article est de présenter les éléments de l'institut ontarien du cerveau à la communauté des sciences neurologiques : la motivation de sa création, la philosophie qui la sous-tend, les principes guidant son développement, l'évolution rapide de sa structure fonctionnelle, les outils disponibles pour atteindre cette vision et la structure de direction pour assurer la réussite. L'objectif unique de la province et de l'Institut ontarien du cerveau est un système global qui permet l'inclusion de la recherche fondamentale dans le système clinique et qui facilite le développement de produits pour bénéficier encore plus rapidement à la santé et à l'économie de la santé : la science avec un impact.

doi:10.1017/cjn.2014.36

Can J Neurol Sci. 2014; 41: 683-693

\section{Motivation}

One in three individuals will be directly affected by a brain disorder in his or her lifetime. In the Province of Ontario, with a population of 13 million, approximately 1 million are currently living with a brain disorder. ${ }^{1}$ The personal, social, and economic costs for affected individuals and their families and caregivers are unquantifiable. Measuring the economic burden for society is a challenge. It includes the direct health care costs and the indirect and opportunity costs of work missed by patients or their informal caregivers. A recent study on the economic toll of brain disorders in Europe found that indirect costs of brain disorders far exceed the direct costs of health care and treatment, more than for any other somatic disease. ${ }^{2}$ The direct and indirect costs in Ontario of mental health and addictions alone is estimated at \$39 billion a year. ${ }^{3}$

The pressing need cannot be denied-but should a province of less than 15 million people make a focused investment? What could Ontario do differently in science that would make a substantial change for its citizens as well as individuals throughout the world? This is the question Ontario asked itself-is it fiscally responsible, and is there sufficient potential of true health and economic impact, for Ontario to tackle the problem of brain disorders?

In response to a study to assess the quality of brain research in Ontario, and Ontario's capacity to respond to this pressing need (The Ontario Brain Institute: A Proposal to Mobilize Ontario's Excellence in Brain Research), ${ }^{4}$ on November 15, 2010 the government of Ontario provided Ontario Brain Institute (OBI) with $\$ 15$ million in start-up funding for a three-year "proof-of-principle" period, with the requirement of raising an additional \$5 million to enhance neuroscience activities in the province.

The first strategic implementation plan set out three priorities to create a system of research, translation, and innovation:

1. Create a patient-centered research system to drive discovery;

2. Engage industry to drive economic results; and

3. Drive borderless knowledge exchange between researchers, patient communities, policy makers, and industry.

\section{Core Principles}

To fulfill this strategic plan, we established core principles. The excellence already existing in Ontario was a major reason for

From the Ontario Brain Institute, Toronto, Ontario, Canada.

Received June 25, 2014. Final Revisions Submitted August 6, 2014 Correspondence to: Donald T. Stuss, President and Scientific Director, Ontario Brain Institute, 438 University Avenue, Suite 1618, Toronto, Ontario, Canada M5G 2K8.

Email: dstuss@braininstitute.ca. 
the creation of OBI, and scientific excellence had to remain the primary principle. This was the fountain of knowledge for improving brain health. Quality of the research in relation to international standing and impact would be monitored ongoing, and evaluated by an independent panel every four to five years before renewal.

The primary focus would be on brain health. To respond to the call from the original report to have impact, the needs of the patient and caregivers (improved diagnoses, better treatments, increased involvement from industry to create appropriate and needed products) would drive outcomes. Therefore, patients, caregivers and their advocacy groups would be involved at all levels of OBI activities as a necessary condition.

After the report, partnerships became our mantra-maximizing the strengths through connections and breaking down silos: between clinicians and researchers, between institutions, between industry and researchers, between patient advocacy groups and researchers, and between disciplines and methodologies. Stated positively, the maximum value of Ontario's neuroscience strength would be realized through integrating and connecting the strengths and through sharing data and their sources.

To maximize the investigation of mechanisms of disease and understanding the heterogeneity of disease etiology and expression, proposals were encouraged that not only integrated institutions and disciplines but also reached across diseases. As an example, the neurodegeneration workshop focused on Alzheimer's disease, Parkinson's disease, amyotrophic lateral sclerosis disease, frontotemporal lobar dementia, and vascular cognitive impairment. This type of cross-disorder, multidisciplinary collaboration was an important first step in our role as catalyst, integrator, and innovator.

The implementation of this partnership model became key. How does one achieve partnerships; how does one tear down the silos; how does one strengthen and speed the implementation of basic science knowledge creation into practice? The answer was knowledge exchange. The most direct way to maximize this communication was through workshops, during which all partners would be present. These workshops were held to initiate the research and are held annually to ensure open exchange, excellence of science, and to facilitate impact.

Brain research has a different quality in that individuals with brain disorders often live longer, creating lifetime impacts on all aspects of the individual and their family. This suggested that the area of contact with these individuals - the hospitals and clinics and patient advocacy groups - should be at the core of our efforts. At least in the early stages, and considering the limited funding, the primary role of OBI would be to facilitate, energize, catalyze, integrate, and connect the clinical and research strengths across Ontario.

To achieve integration and partnerships, and to realize longterm objectives that could be derived from a longitudinal approach, OBI would not be a typical granting agency. Rather, it would provide sustained, research program-based funding providing that the programs maintain scientific excellence and reach the agreed upon milestones. The primary use of the funds would be to fill in the gaps in the system to enhance collaboration and the translation of research. For example, funding might be used to provide clinician release time to ensure clinical involvement and enhance patient recruitment across the province or hire coordinators to ensure common standards of training for assessments and maximize integration. This maximized the number of individuals with disorders who might participate in a study (i.e. increased sample size) and the sharing of data, while also providing some consistency of funding. In this way, stable and enduring collaborations could be forged.

Translation of the research into useful products and therapies is an important means of impact. Neuroscience industries became our core partners and began attending our workshops. To maximize this success, our funding model was set up to not take ownership of any intellectual property created by our research programs. It does, however, encourage institutions to commercialize research by working to enhance valuable partnerships.

Working together in a province-wide manner creates the possibility of enhanced productivity and impact. To ensure this, it was important that all data be gathered in a standardized manner. This results in comparability of data and enhanced sample size for any study. And in just achieving this goal, patients across the province involved in the programs would benefit from "research quality" care." 50 maximize use of these data with the realization that the data are gathered with public funds, an informatics platform to collect and archive the data for current and future use was necessary-this resulted in the development of Brain-CODE, the Centre for Ontario Data Exploration (see the following section).

Finally, to ensure excellence we created both a Science Advisory Council and an Industry Advisory Council (Appendix A) to ensure that the selected programs focused on excellence and impact. We also established international advisory committees for specific roles of OBI, such as evaluation and informatics.

\section{Deciding the Content of Research}

\section{Identifying priority research themes}

The outcome of the initial "Talk and Listen" tour in early 2011 was 33 letters of intent covering a wide range of brain disorders. In line with our vision of integration and collaboration, the letters of intent that passed the threshold after external review were consolidated through discussions and seven were approved to prepare a full application. Organized province-wide workshops for these seven disorders were held to develop the full applications for review.

With a total investment of $\$ 15$ million over three years from the Province of Ontario, and even with the requirement to raise an additional one-third of the funds to support the entire enterprise, not all seven programs could be funded. Given OBI's proof-of-principle mandate, and a relatively short time-frame to show impact, the review process favored programs that were most ready to benefit from our approach, meaning that integration of excellent research within the clinical framework, and avenues for commercialization, knowledge exchange, and ultimately early patient impact had to be clearly in place. Based on external review, three applications were initially funded from the seven: neurodevelopmental disorders (including autism, attention deficit hyperactive disorder, obsessive compulsive disorder, and intellectual disability), cerebral palsy, and epilepsy. These three programs are now called, respectively, POND (Province of Ontario Neurodevelopment Disorders), CP-NET, and EP-LINK. 
A full independent review of OBI was held in October 2012 (the end of the three-year proof of principle was March 31, 2013; a review and resubmission was therefore needed in the fall of 2012), examining the success and potential of the endeavours. Notwithstanding the difficulties of the start-up phase, the reviewers concluded:

\begin{abstract}
"It is clear that the OBI has galvanized the scientific research communities among different thematic areas and neuroscientists in Ontario. This is seen, in particular, in the funded Integrated Discovery programs, where groups of researchers and clinician-scientists have come together, on a scale and scope not previously realized. This organizational effort in connectivity alone has led to new connections among the community that is producing novel high quality research groups with the potential for high impact research teams in the future."
\end{abstract}

External review, 2012

\section{Further Evolution}

Based on this review and the success of the initial three programs, OBI submitted a renewal application in the fall of 2012. In January 2013, the government announced $\$ 100$ million for a five-year period, with specific conditions on maximizing partnership and impact. Again, after review, our Board extended the funding of the first three programs, and awarded funding for two additional programs - depression and neurodegeneration. These two new programs are called CAN-BIND (Canadian Biomarker Integration Network in Depression) and ONDRI (Ontario Neurodegenerative Disease Research Initiative), respectively.
With this renewal came the creation of a permanent Board of Directors. General descriptions of these five programs, including links to their individual websites, can be found on the OBI website (http://www.braininstitute.ca/research).

\section{EvOLUTION-How OBI WoRKS}

\section{An integrated system to improve patient care and drive economic development}

Our second strategic plan, now for five years, formalizes the methods of operations that OBI had been evolving in its first phase (Figure 1).

OBI is structured to embed basic science discovery into the clinical framework and develop new products to improve health as soon as possible-science with impact. Each component of the system depicted in Figure 1 plays a specific and crucial role, but interactions are not linear-knowledge flows in many directions. The management of OBI mirrors the structure of the system, each having a team to ensure excellence, impact, and integration as needed to maximize output.

\section{Partnerships}

The research programs that we fund enable clinicians and hospitals, academic researchers and institutions, patient advocacy groups, and industry partners to work together in an integrated manner. The methods by which this occurs have been described previously. Partnerships and coordinated approaches have been successfully developed across Ontario. The first three funded programs involved more than 80 researchers, 35 companies, and 20 patient advocacy groups. With the current complement of five programs, this has increased to more than 200 researchers

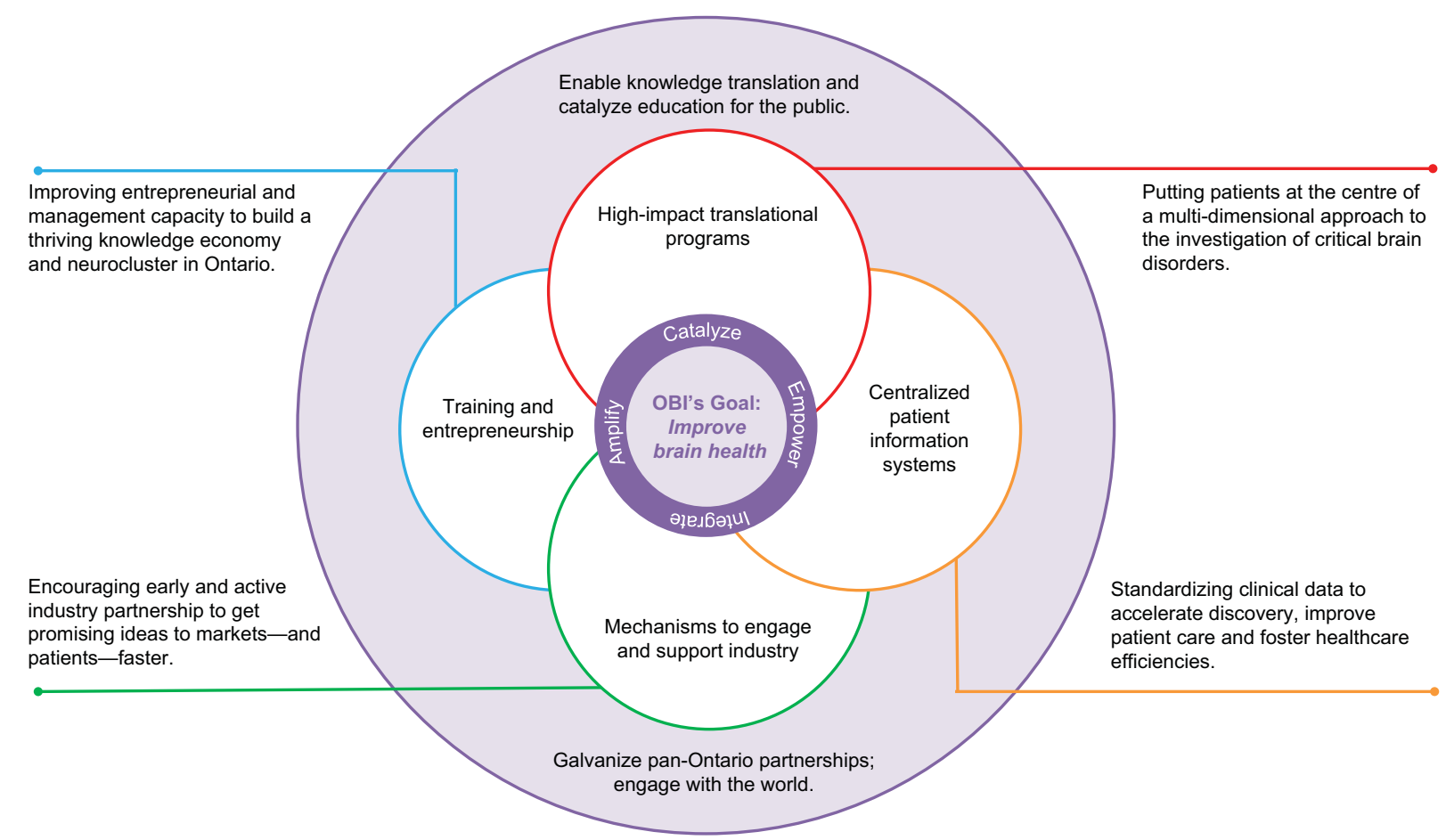

Figure 1: The OBI system. The four pillars of OBI's system drive improvement to individual brain health. 
and clinicians, 45 companies, and 29 patient advocacy groups and service providers. Each research program has a Patient Advisory Committee, with representation from patients and patient advocacy groups. With growth and stability, partnerships will extend globally to maximize research and impact. The number of individuals with disorders involved in the research programs will exceed 5000 by 2018 . This network is depicted in Figure 2.

\section{Research programs}

Our programs are structured to allow the development of new questions and new approaches, enhance clinical impact, and foster the integration of patients and industry to rapidly translate discovery into impactful products. The term "Integrated Discovery" describes an approach to research that is truly transdisciplinary involving the collection of different types of data, ranging from genetic and molecular to imaging and behavioural. A major assumption underlying this approach is the heterogeneity of neurological and mental health disorders. Comorbidities may affect different disease processes differently. There may be interactions between common underlying characteristics at the genetic, proteomic, biochemical levels, as illustrated in Figure 3. The grouping of programs by symptom-based disorder accepts the existence of broad categories of disorders to guide research (Figure 4). At the same time, we are cognizant of the potential of finding new research-based classifications based on the information being gathered in the Integrated Discovery programs (New York Times, February 3, 2014, interview with Dr. Thomas R. Insel).

There is a common approach to how data are collected, both within a disorder and, to the extent possible, across disorders. Assessments are standardized, including the clinical evaluations, the selection of measurements done through workshop discussion. Regular training is completed to ensure quality of data throughout the province. This allows data to be shared across the province, maximizing clinical impact.

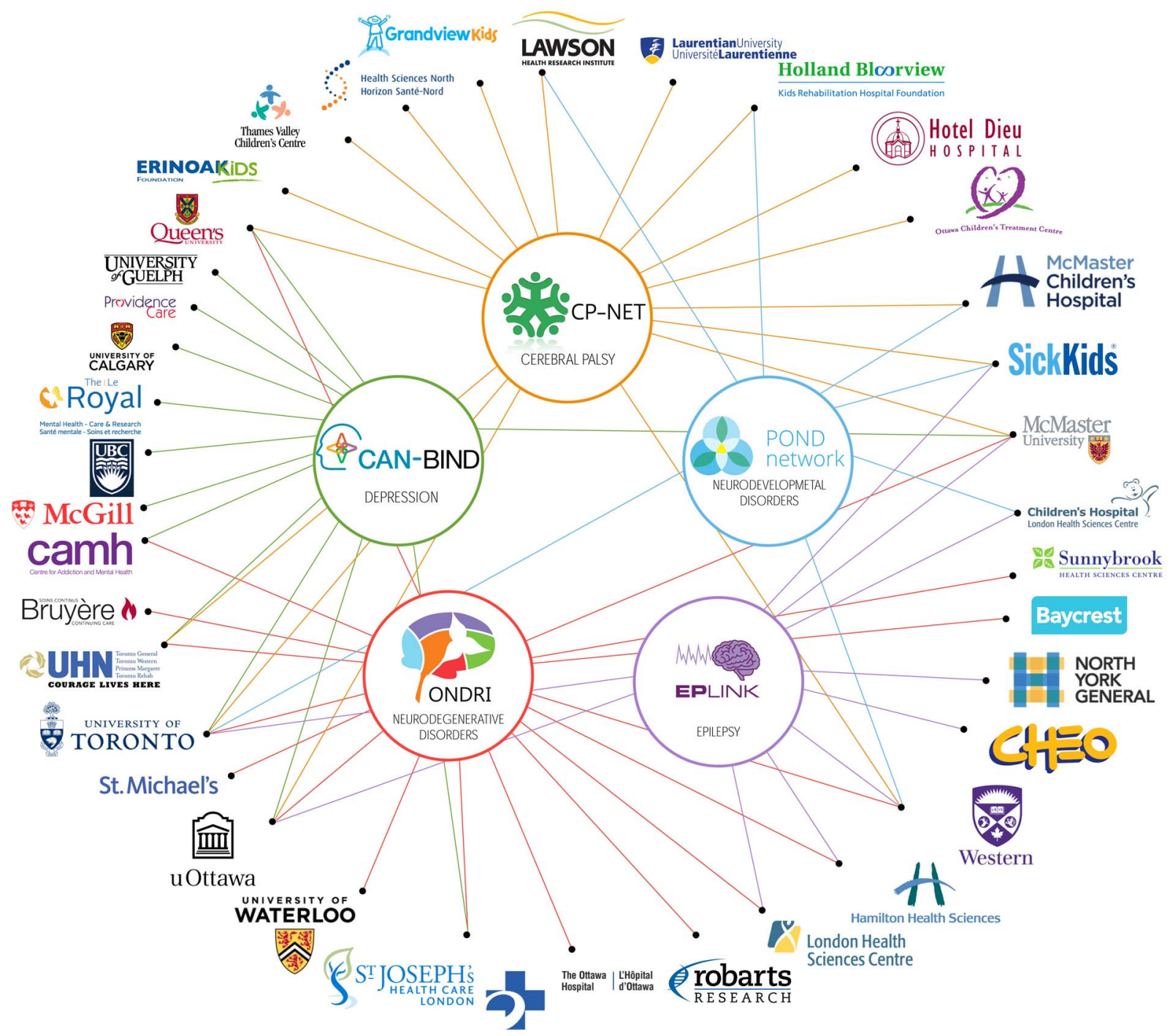

Figure 2: Research partnerships. Through the five Integrated Discovery Programs, OBI is engaged in partnerships with 37 institutions to form a network of research collaboration across Ontario. 


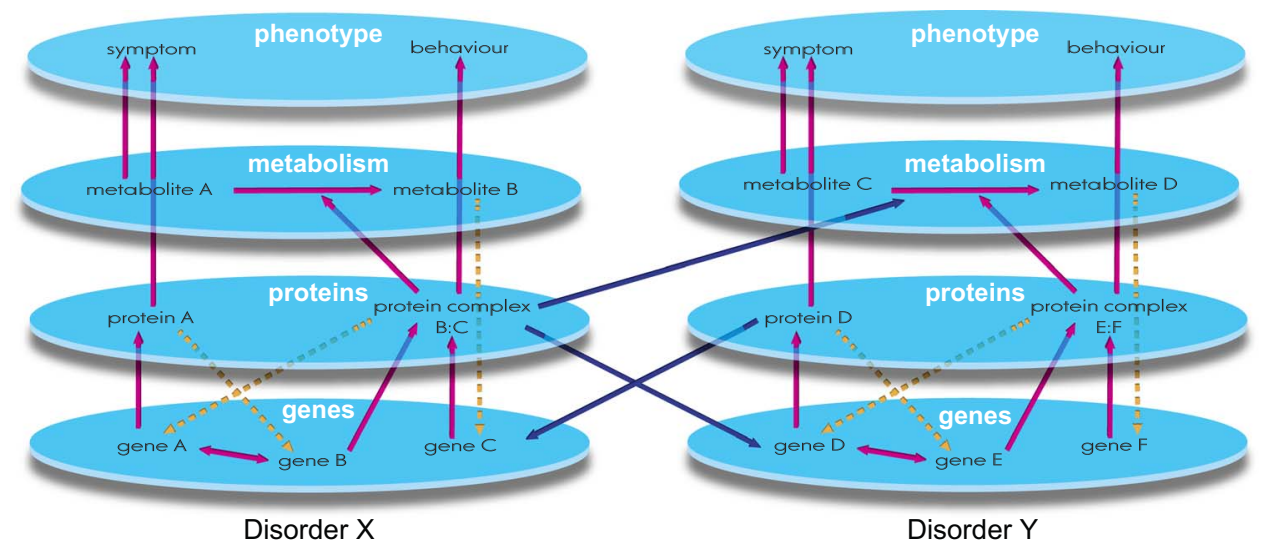

Figure 3: Layers of interaction and the complexity of brain disorders. Complex interactions occur within and across the layers of biology at the levels of genes, proteins, and metabolism that give rise to the symptoms, behaviours, and phenotypes observed in brain disorder X. In many brain disorders, overlap also occurs between underlying factors of different disorders as illustrated by the cross-talk of genes and proteins shared by disorders $X$ and $Y$. This complexity and overlap necessitates a research approach set up for study both across the layers of biology and across the disorders. This approach facilitates the study of mechanisms affecting disease expression, with the hope for development of targeted treatments.

An excellent example is imaging. Despite the obvious challenges of this undertaking, such as the variability in technology and expertise among the institutions involved, a standardized acquisition sequence across Ontario is being established within Integrated Discovery programs as feasible (sequences may be different for younger and older populations). Harmonization to set the standards is being achieved by having one group of individuals scanned across the scanners being used in the Integrated Discovery programs. Maintaining quality of scans is achieved by a commonly used phantom brain procedure. Through the process of standardization and exercises in teaching, learning, and sharing, we have increased the imaging capacity and enhanced the research potential of the province. If all imaging sites are standardized within at least one Integrated Discovery program, then we have the ability to prospectively monitor and catch scanner changes that can benefit all programs using that scanner.

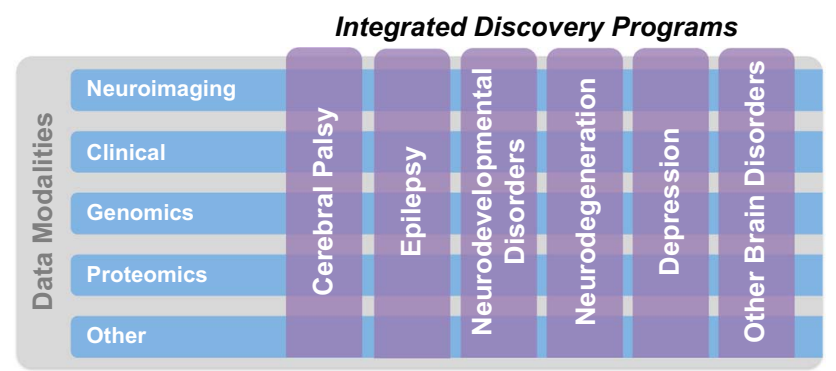

Figure 4: Integrated Discovery approach. The integrated approach to research requires that standardized neuroimaging, clinical, genomic, proteomic, and other data are captured from the five research programs in brain disorders: cerebral palsy, epilepsy, neurodevelopmental disorders, neurodegenerative disorders, and depression. This facilitates innovative hypothesis-driven investigation and data-driven discovery approaches both across data modality and across disorders.
Detailed characterization of patient cohorts provides several benefits, such as enabling larger scale analysis of genetic characteristics of subgroups of individuals with a disorder, and providing the potential for targeted cohort-specific, earlier-phase pharmacological trials. With in-depth information on individuals with a specific disorder, researchers will be able to take a new look at root causes among diverse disorders. Because the approach is part of a province-wide system where clinical and research data are gathered and kept in a common format, it also provides the opportunity to apply findings from one disorder to others, and integrate knowledge if individuals have more than one disorder. Not least, the Integrated Discovery approach is an opportunity to study mechanisms of disease across disorders.

As such, the approach is hypothesis-driven, yet allows exploratory, curiosity-driven research both currently and in the future. In the development of OBI, we realized very early on that maximizing the value of the Integrated Discovery approach to research requires a state-of-the-art informatics platform.

\section{Centralized Patient Information Systems}

\section{Managing and amplifying impact of the data}

With the creation of Ontario-wide research programs, the necessity and opportunity to create a virtual center for data sharing and exploration quickly became apparent. With limited funds to develop a platform "de novo," we sent out a request for proposals seeking to build an informatics platform based on existing resources. The successful applicant was a consortium called InDoc, a group of not-for-profit organizations that, by working together, brought expertise in molecular data collection, imaging, security, military-grade storage, computing, and informatics (see http://www.braininstitute.ca/brain-code for more details). The platform was developed taking advantage of databases and computing platforms funded by previous government initiatives that were focused on building computing and networking capacity in Ontario. As with all our initiatives, the development of this informatics platform is guided by an International Advisory 
Committee (see Appendix) that provides expertise and direction on how to ensure that Brain-CODE continues to meet international best practices on data organizing, sharing, and analysis.

The platform, called the Brain-CODE, is a virtual space for researchers to share and analyse their data. The tool itself is an extensible neuroscience informatics database that manages the collection, storage, and analysis of multidimensional data collected from patients with a variety of brain disorders participating in our Integrated Discovery programs. Using Brain-CODE, researchers can share their discoveries and begin looking across disorders and brain conditions to better understand common underlying causes.

Several characteristics are necessary to allow Brain-CODE to achieve its potential. The first is to ensure the highest standards of privacy and security. The data in Brain-CODE are housed at Ontario's High Performance Computing Virtual Laboratory at Queen's University, which is the only facility of its kind in Canada supporting regulatory-compliant processes. We worked closely from the beginning with the Information and Privacy Commissioner of Ontario to ensure the platform is secure and compliant with regulatory requirements; this led to the designation as "Privacy by Design" ambassador. Brain-CODE features a unique, three-zone security platform that allows for tiered access to the data.

Privacy is embedded within the design of Brain-CODE through the following: patients must provide informed consent - consent forms outline how the data will be used; health card numbers are collected but encrypted at the time of capture and encryption is validated before data are transmitted; and methods are in place to identify direct identifiers for processing and removal where there was no approval to collect them. Zones provide the opportunity for tiered access. Only personal health information relevant to project needs is gathered, and this is available only to researchers with access to zone 1 , which is the project space for the authorized users for that project. Data in zone 3 , available to researchers across the world under specific conditions, have gone through the deidentification process. Before the release of any data, a Privacy Risk Assessment Tool is used to assess the risk of any data being reidentified. Brain-CODE is designed to allow secure linkages to other databases, allowing for a richer level of analysis.

A cornerstone of effective collaboration among researchers involves the sharing of data. For researchers to be able to share and analyze their data properly, they must be comparing apples to apples - the data must be standardized. The standardization of data collection on a province-wide scale, across several research platforms - and including multiple data types-is a defining feature of Brain-CODE. Efficient comparison of data, both within the province and with external federated databases, is maximized when data are in the same format.

Common Data Elements (CDEs) help define and format the different types of variables that researchers analyze. OBI researchers and expert panels establish the most appropriate CDEs through a consensus process. A select number of CDEs will cross all disorders, providing effective comparison across disorders and databases, and allowing the evaluation of comorbidities.

\section{Potential impact of the structure of the Integrated Discovery programs and Brain-CODE}

Brain-CODE is necessary for the research programs to reach their full potential. It augments the value of the research program data by linking with other databases and leveraging the existing resources. This provides the potential for richer analytics. In addition to amplified research outcomes, the potential links with health administration data, possible in the Canadian health system, provides opportunities to evaluate cost of service delivery in well-characterized patients, and to initiate discussions on service delivery.

For example, we are beginning a pilot linkage between a dataset from our epilepsy research program and the Ontario health administrative data held by the Institute for Clinical and Evaluative Sciences. This linkage will enhance the outcomes of our clinical study by allowing the researchers to look at the impact of the treatment, both on the health of the individual, and the health care system. In parallel, we will be able to get information on both the efficacy of a novel treatment and the potential cost-savings to the healthcare system.

\section{COMMERCIALIZATION AND ECONOMIC IMPACT}

Commercialization is a critical step in translating research ideas into better patient care. Our model of commercialization is neither push nor pull; partnerships are at the root of this translation. This again is maximized through knowledge exchange and facilitating a dialogue between the private sector and not-forprofit institutions from the beginning. Researchers continue to focus on science; industry can indicate the most important needs, share their knowledge and resources on a precompetitive basis, and be opportunistic in identifying commercializeable products early in discovery.

A major objective of these efforts is to create an Ontario neurotechnology and therapeutics neuroscience cluster. From a commercialization perspective, a cluster identifies a geographic concentration of companies, suppliers, and service providers of a related industry all within a "commutable distance." Clusters allow companies to maximize their efficiency by providing for greater sharing of local infrastructure, better matching of skills between employees and employers, and the diffusion of knowledge among workers.

In 2011, a study was commissioned to identify Ontario's potential for a cluster. Although the results suggested significant promise, it pointed out two opportunities that must be seized to ensure success: developing access to capital and cultivating management talent.

OBI's catalytic role is to seek out funding to support Ontario researchers and companies. In addition to an effort to find and connect companies to researchers and to assist in finding funds to support such efforts, OBI received a grant from the government of Canada's Federal Economic Development Agency for Southern Ontario (FedDev Ontario) to create NeuroTech Ontario. FedDev Ontario approved an investment of nearly $\$ 11$ million matched by more than $\$ 11$ million in private-sector investments for a combined total of greater than $\$ 22$ million for the development of NeuroTech Ontario. This program is aimed at developing new devices and treatments for brain disorders. Launched in 2011, the program has already launched three new products, led to ten intellectual property disclosures, and created more than 100 jobs. 
One example is the development of an online learning platform called Thriver that will be used by parents and educators to assist in the cognitive development of children through gaming. This product was borne out of a partnership between the University of Windsor and Online Teacher Education Portal (OTEP Inc.) and is currently being implemented by the YMCA Academy, a high school for students with learning disabilities.

\section{Cultivating the Right Talent}

Cultivating management talent to drive a successful cluster in the neuroscience commercialization sector was the other opportunity identified in the report. Ontario does have a growing pool of highly qualified neuroscientists whose skills are needed to sustain a neuroscience cluster in Ontario, but their potential requires the right catalyst and appropriate opportunities. OBI decided to build capacity for a knowledge-based economy in Ontario; create expertise in the management of neuroscience research, knowledge translation, and commercialization of neuroscience; and increase employment options for neuroscientists that contribute to a knowledge-based economy. We called this the Experiential Education Initiative, and it currently includes an Entrepreneurs Program, a Graduate Opportunity Internship Program, and a Graduate Opportunity Management Fellowship Program. Each has a specific program of mentoring and guidance.

\section{Entrepreneurs Program}

The aim of this program is to drive the emergence of entrepreneurial spirit in the Ontario neuroscience cluster, increasing both the number of entrepreneurial scientists and, ultimately, the number of neuroscience-related companies. The program provides $\$ 50,000$ to help postgraduate students in neuroscience or related fields to focus full-time, over a one-year period, on commercialization activities related to a neuroscience-based opportunity. The program offers mentorship, networking, incubator space, training through University of Toronto Early-Stage Technology (UTEST) and others, and links to local innovation hubs. Funding partners include the Ontario Centres of Excellence, and training partners include The Impact Centre, Lead to Win, and TechAlliance E 1.0 program. In the first two years of the program, the 15 entrepreneurs have secured more than $\$ 4.3$ million in follow-on funding for their companies, built 26 prototypes, and filed 13 patents or disclosures.

\section{Graduate Opportunity internships}

This program was developed to offer opportunities away from the bench to graduate neuroscientists. We aim to place up to 10 interns annually, with the assistance of leveraging partnerships such as FedDev Ontario's Graduate Enterprise Internship and the Ontario Centres of Excellence. To date, this program has placed 19 interns, leveraging more than $\$ 290,000$ from partners and participating companies. These placements may be with small- to medium-sized enterprises, large multinationals, or commercialization organizations across the province. Our partners provide cofunding and subsidies for company contributions to place highly trained graduate students in new and exciting career opportunities that have led to a greater than $90 \%$ employment rate for individuals coming through this program.

\section{Graduate Opportunity Management Fellowships}

The Management Fellowship program aims to develop the next generation of leaders in the not-for-profit sector in Ontario. The goal of this program is to develop business acumen and management skills to broaden the career path for recent neuroscience graduates. The Fellowships provide hands-on experience away from the bench in the areas of research management, commercialization, knowledge translation, governance, and policy. In this program, the Fellows are embedded within one of our teams for six months followed by another six-month placement at an external partner organization. This program has been supported by funding support from the Innovation Institute of Ontario.

Collectively, the three programs of the Experiential Education Initiative have made great strides to help Ontario's highly trained neuroscience graduates move beyond the bench and integrate into the Ontario neuroscience cluster. For example, two of the entrepreneurs are validating their neurotechnology devices through our research programs; a management fellow and three interns have been placed at knowledge translation partner organizations; a management fellow will be working with the InDOC Consortium, the group developing BrainCODE; ten interns have been placed with industry partner organizations including Industry Advisory Council member companies, companies started by our entrepreneurs, and member companies of NeuroTech Ontario.

\section{Integrated oversight}

Success in these endeavours requires expertise, regular review, and oversight. In addition to the Industry Advisory Council described previously, OBI created the Ontario Brain Innovation Council, an integrative structure to engage the community and oversee the development of the cluster. Members derive from industry, research, government, finance, and patient advocacy groups. Ontario Brain Innovation Council directly and indirectly supports the OBI's current cluster programs by helping to create new relationships between existing players, recruit and develop new talent, advise on efficient use of resources, share best practices, and to identify new market opportunities for organizations to work together.

\section{Knowledge Exchange and Communications}

Effective and open knowledge exchange is necessary to drive science with impact. There is a real need for research knowledge (information, discoveries, and ideas) to be made accessible to those who can use or benefit from it. When it is shared through the right medium, to get to the right people at the right time, science becomes impactful. Open communication about the work within the OBI research programs, partner organizations, and building awareness about the brain and brain disorders creates public interest and a fertile environment which is receptive to our knowledge exchange activities. And this engagement creates opportunities to put evidence-informed tools and services into action.

This essential blending of communication and knowledge exchange is what we collectively refer to as Outreach, and serves as a bridge between OBI programs and the community. It allows 
OBI to build, integrate, and engage with our partners through story-telling, connecting people, aligning evidence with care, and evaluating impact.

\section{Telling stories and connecting people}

An educated and informed public is an essential partner to fulfill our overall goal of improving brain health. This involves increasing awareness of brain disorders to promote broader understanding and to reduce stigma, and highlighting neuroscience excellence from the individuals and/or groups across Ontario actively working to improve brain health.

To ensure successful knowledge translation we create frequent opportunities for researchers, clinicians, patients, industry, and other not-for-profits to share this knowledge. We have built an environment that allows these groups to work together more often, and more effectively. This ensures that ideas, discoveries, and communication flow seamlessly both within and between groups and ultimately to the patients and community.

At one of our workshops, a member of the Patient Advisory Committee commented on the need to validate the innovative clinical service they were providing to individuals with the disorder. An active researcher said this harmonized with their own research interests, leading to an experimental research design to assess the clinical service. Without the opportunity of the knowledge exchange, this would not have occurred.

\section{Aligning evidence with care}

The ultimate goal of knowledge translation is to close the gap between "what we know" and "what we do." This means we need to drive the translation of research into evidence-informed care. With partner organizations, we conduct, support, or initiate the development of small-scale pilot studies focused on addressing unmet patient needs.

In an effort to move knowledge to action and better care, we published a report that analyzed more than 50 years of research and found that being physically active is an effective way to reduce the risk of Alzheimer's disease and improve the everyday lives of those living with Alzheimer's disease. The widespread attention the report received provided an amenable environment for us and several partner organizations to begin exploring ways to implement this evidence and promote physical activity as an effective lifestyle intervention for the prevention and management of Alzheimer's disease. This led to an implementation partnership with the Alzheimer Society of Ontario to increase physical activity in individuals with Alzheimer's disease, and involvement with an Ontario researcher specializing in this area led to a specific research project assessing the value of implementing the program.

\section{Evaluating impact}

The totality of our efforts needs to be evaluated to gauge their collective impact towards the goal of improving brain health.
Evaluation of these activities is necessary to demonstrate return on the investment made by the Ontario government, and to also validate the effectiveness of the system in place at the core of our initiatives. Our role as a catalyst is difficult to measure, which is why we are guided by the recommendations and expertise of our Evaluation Advisory Committee. We are actively working with them to embed evaluation metrics, including both qualitative and quantitative measures, into our work. No single activity will demonstrate our overall goal, which is to improve brain health. Instead, it will be the aggregate of our total efforts, as measured in both numbers and stories that will indicate our patient impact and improvement in the lives of those individuals living with brain disorders.

\section{Management Structure to Ensure Success}

The integration of resources and the establishment of a structure to maximize impact are required by virtually all funding agencies. Grant applications request plans for commercialization, knowledge translation, and patient involvement. OBI's role as a "hub" takes responsibility for success in these domains by having teams work with the researchers, forging connections that may not be easy for researchers and clinicians. For example, OBI staff has formed Patient Advisory Committees for all of the research programs, including having all of the advocacy/patient groups meet together annually. The commercialization team searches for possible collaborations of researchers with companies, especially at an earlier stage. The Brain-CODE group is investigating not only maximum privacy procedures, but also determining the status of analytics capacity locally and internationally and helping to ensure our researchers have that capability available to them.

Each research program has two focused innovation teams, for science and commercialization, mirroring the structure of the broader Science and Industry Advisory Committees. These smaller groups, with expertise directly related to the disorder being studied, are called the Science and Core Innovation Teams. Membership on these Innovation teams includes representatives from the Science and Industry Advisory Committees, ensuring open communication. These innovation teams meet with the research program at the annual workshop. They review the success in meeting milestones established the year before, provide mentoring and advice both on important international science trends and derived from their own expertise, and suggest commercialization opportunities.

Continued funding is dependent on achieving impact. An evaluation process is being established, again with an international advisory committee, to have objective measures of all OBI activities. Every five years, each research program will be evaluated by an international external review team.

\section{How does it work?}

To achieve these goals, particularly in the tight timelines, the management team has developed a style of functioning to ensure success in its primary roles as catalyst and integrator. Top quality work is the ultimate goal. Against this backdrop, in many regards the style may be described as "start-up," with an emphasis on speed, efficiency, and agility. The mind-set is "do it," not just "say it"; for example, being patient-centered means having patients, advocacy groups, and health charities involved from the beginning. OBI staff facilitates success in commercialization, health 


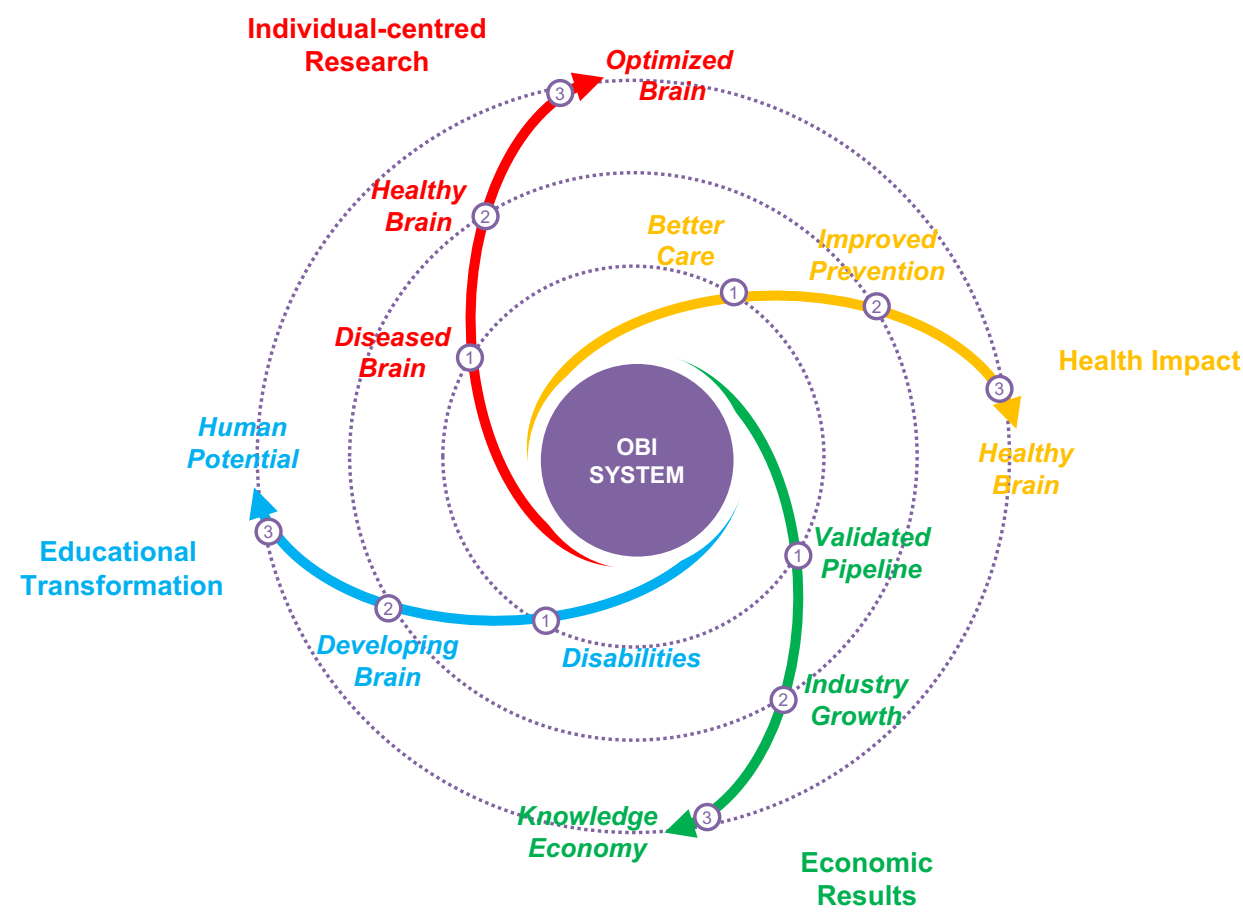

Figure 5: The three phases of OBI development. Mapped out as a long-term plan, OBI's system achieves enormous educational, economic, and health impact as the three phases are implemented. OBI is applying its system to projects across the three phases to deliver scientific, health, economic, and educational impact. This schematic provides a model for visualizing the contribution of each of the three phases, shown as numbered rings: (1) Investigating Disorders of Brain Functioning, (2) Understanding Healthy Brain Functioning, and (3) Optimizing Brain Performance. The spiraling arrows chart a path through the outcomes of each pillar to the four dimensions of impact.

impact, and uptake of new knowledge. Establishing milestones and evaluating success (or identifying failure) is built into the very fabric of all activities. Public trust means building on other past and current investments, public and private, to provide maximum value for the dollar; public engagement ensures involvement from the public in research projects and adoption of new knowledge.

\section{WHERE NeXT?}

The proposal to the government in 2012 for the renewal of funding for the OBI outlined a possible long-term vision in brain research. The OBI system of innovation has the potential to drive an initiative much larger than what had been originally set out in the OBI's strategic plan (Figure 5).

Understanding brain disorders represents a critical component of the OBI's first phase, but brain health is not simply about the treatment and prevention of disease; it's also about supporting and maintaining normal development and optimizing how we use our brains early in life as well as enhancing the performance and wellbeing of our brains throughout the entire lifespan. This critical point is what drove the proposal for three distinct phases of development: disorders of brain functioning; understanding healthy brain functioning; and optimizing brain performance. Each has brain health as its core; each should be structured to have impact not just in new knowledge, but also in health, economic results, and maximizing potential through education. The ultimate goal is creating a knowledge-based province based on understanding and maximizing brain functioning not only for each individual, but also for society as a totality.

\section{So What Is the Value of This Approach-That Is, So What?}

In one sense, the OBI approach has the same goals as all agencies, researchers, clinicians, patient advocacy groups, and administrators have-excellent research that has both intrinsic (the creation of new knowledge) and applied (short- or long-term) value. We focused our efforts on the creation of a system to catalyze and facilitate the achievements of all of the goals. In Figure 1, the large circle encompassing all activities illustrates the simultaneous nature of the activities rather than linear development. The use of overlapping inner circles symbolizes the integration of all facets of research activities: research itself; the use of data via informatics and analytics; obtaining the maximum value of research through development of products and economic impact; and outreach through knowledge translation and implementation. We sometimes describe it as embedding research into clinical practice and care, and embedding industry development into research, through partnership with a view to implementation (knowledge translation).

There are specific benefits to functioning as a system. The integration of all partners from the very beginning of research hastens and facilitates the implementation of discovery and the creation of new health products. 
The standardization of assessments and the sharing of data have many positive outcomes. Assessment standardization in all of the clinical centres involved in the research means consistency of clinical evaluation at a research level across the province, with subsequent equalization of clinical care. The number of individuals involved in research activities is increased through the province-wide approach; this sample size increase has obvious benefits for research power. With a greater number of individuals involved, and the careful standardized characterization, there is increased opportunity to observe the variability and heterogeneity of disease expression, ${ }^{7,8}$ with the potential benefit of targeted pharmacological and behavioural treatments and the study of mechanisms of disorders across diseases. The detailed characterization of individuals studied should be attractive to pharmaceutical companies interested in studying the specific efficacy of disease. In essence, there is a real opportunity for what is called "personalized medicine."

In earlier versions of Figure 1, we used the label "OBI Innovation System." We have now decided to minimize the use of the word "innovation." The term is difficult to operationally define and it sometimes seems using the word is considered as good as being "innovative." What OBI is doing is simple and "old school"; the difference is in ensuring implementation within a flexible open system. Our hope is to establish a system where basic science flourishes because of patient characterization and removing boundaries between diseases to facilitate studies of mechanisms of disorders; where the informatics platform and data sharing within and across diseases provide an opportunity not only for hypothesis driven research, but also for the chance finding, data mining, and the creation of new hypotheses; where discovery and treatment are more closely linked; where industry works closely with researchers to implement their discoveries into new products; where new products for improved patient health have an economic benefit through new companies and jobs; where the creativity of the researchers and the needs of individuals with disorders fuel new research questions and ideas; and where the network of patient advocacy groups and health charities as well as knowledge exchange with primary health care givers push early and rapid uptake of new diagnoses and new treatments.

\section{Behind the Curtain}

There are stories and greater content behind each of the sections described briefly here. These can be found in our videos, newsletters, reports, all of which are on our website.

\section{ACKNOWLEDGMENTS}

The creation and evolution of OBI rests particularly on the following: Joseph Rotman, current Chair of the Board of OBI and primary mover for the integration of commercialization into $\mathrm{OBI}$ and Joseph Martin as cochair of the study team; R. Murphy and F.I.M. Craik for their foresight in their document arguing for the creation of $\mathrm{OBI}^{4}$ and to those who provided them with feedback and advice; B. Miller and M. Noble for their enormous roles in the development in the initial stages. The author is also indebted to the Strategic Health Initiative, especially Ryan Wiley, for assistance in developing the strategic plans; C. Stiller, M. Noble, and K. Knox for ongoing advice; members of the OBI Outreach team (K. Nylen, J. Antflick, M. Wilson) for assistance in writing and forming this article, and all OBI staff for their input; to all OBI staff for making OBI actually function as described; to our Board and Advisory Councils for advice and guidance; and M.P. Alexander for his creative editorial input.

\section{REFERENCES}

1. Institute for Clinical Evaluative Sciences/Public Health Ontario. Opening Eyes, Opening Minds: The Ontario Burden of Mental Illness and Addictions Report. 2012.

2. Wittchen HU, Jacabi F, Rehm J, et al. The size and burden of mental disorders and other disorders of the brain in Europe 2010. Eur Neuropsychopharmacol. 2011;21:655-79.

3. Ministry of Health and Long-Term Care. Every Door is the Right Door. Towards a 10-Year Mental Health and Addictions Strategy A Discussion Paper. 2009.

4. Ontario Brain Institute. The Ontario Brain Institute: A Proposal to Mobilize Ontario's Excellence in Brain Research. 2010.

5. Kryzanowska MK, Kaplan R, Sullivan R. How may clinical research improve healthcare outcomes? Ann Oncol. 2011;22(supplement 7): vii10-5.

6. West J, Wright J, Tuffnell D, Jankiwica D, West R. Do clinical trials improve quality of care? A comparison of clinical process and outcomes in patients in a clinical trial and similar patients outside a trial where both groups are managed according to a strict protocol. Qual Safety Health Care. 2005;14:175-8.

7. Stuss DT, Binns MA. The patient as a moving target: The importance to rehabilitation of understanding variability. In Stuss DT, Winocur G, Robertson IH editors. Cognitive neurorehabilitation. 2nd ed. Evidence and Application. Cambridge: Cambridge University Press; 2008, pp 39-61.

8. Gerogiades S, Szatmari P, Boyle M, et al. Investigating phenotypic heterogeneity in children with autism spectrum disorder: a factor mixture modeling approach. J Child Psychol Psychiatry Allied Disciplines. 2013;54(2):206-15.

\section{APPENDIX: OBI'S ADVISORY COUNCILS, GOVERNANCE, AND ADVISORY COMMITTEES AS OF MAY 2014}

\section{Science Advisory Council}

Marcus E. Raichle (Chair), Washington University in St. Louis; Joseph T. Coyle, Harvard University; Fred H. Gage, Salk Institute; John Hardy, University College London; Daniel S. Marcus, Washington University in St. Louis; Joseph B. Martin, Harvard University; Richard Murphy, Richard Murphy \& Associates; William J. Powers, University of North Carolina; Trevor Robbins, Cambridge University; Olaf Sporns, Indiana University; Brian A. Wandell, Stanford University.

\section{Industry Advisory Council}

Todd Vienneau (Chair), GlaxoSmithKline Inc.; Anne VivianScott, BKIN Technologies; Doron Sagman, Eli Lilly Canada Inc.; Genevieve Lavertu (Greg Molnar), Medtronic of Canada Ltd.; John Andrews, Neuraxon; John Soloninka, Health Technologies Exchange; Rosemarie Childerhose, Valeant Canada LP; Lisa Wilcox (Mark Lundie), Pfizer Canada Ltd.; Pat Horgan (Don Aldridge), IBM Canada Inc.; Cynthia Stewart, GE Healthcare Canada; Alison McLean, Nestlé Health Science, Canada.

\section{Board of Directors}

Joseph L. Rotman (Chair), Roy-L Capital; Donald T. Stuss, Ontario Brain Institute; Susan Fitzpatrick, James S. McDonnell Foundation; Suzanne Labarge, McMaster University; Jeff Lozon, Revera Inc.; Hugh MacKinnon (Vice-Chair), Bennett Jones LLP; Marcia Moffat, Compassar Solutions; Valarie Pringle, Journalist and Broadcaster; Marcus E. Raichle, Washington University at St. 
Louis; Meredith Saunderson, Not-for-Profit Volunteer; Todd Vienneau, GlaxoSmithKline Inc.; Yuen Pau Woo, Asia Pacific Foundation of Canada.

\section{Brain-CODE International Advisory Committee}

Daniel Marcus, Washington University at St. Louis; Rodrigo Lopez, European Bioinformatics Institute; Charles Peck, Neuroinformatics Consultant; Ann Cavoukian, Information and
Privacy Commissioner of Ontario; Sean Hill, International Neuroinformatics Coordinating Facility, Karolinska Institute.

\section{Evaluation International Advisory Committee}

Martin Buxton, Brunel University; Jonathan Grant, King's College London; Anne-Marie Engel, Lundbeck Foundation; Anas el Turabi, Harvard University; Kathryn Graham, Alberta Innovates Health Solutions; Gary Teare, Saskatchewan Health Quality Council. 